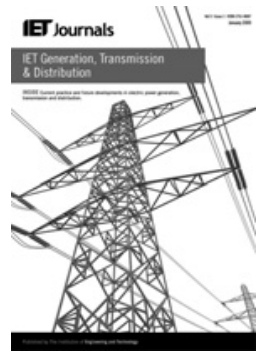

\title{
Multiobjective multistage distribution system planning using tabu search
}

\author{
Benvindo Rodrigues Pereira Junior ${ }^{1}$, Antonio Marcos Cossi², Javier Contreras ${ }^{3}$, \\ José Roberto Sanches Mantovani ${ }^{1}$ \\ ${ }^{1}$ Electrical Engineering Departament, São Paulo State University, UNESP, Ilha Solteira, Brazil \\ ${ }^{2}$ Mathematical Departament, São Paulo State University, UNESP, Ilha Solteira, Brazil \\ ${ }^{3}$ E.T.S Ingenieros Industriales, University of Castilla - La Mancha, Ciudad Real, Spain \\ E-mail: Javier.Contreras@uclm.es
}

\begin{abstract}
This study presents a multiobjective tabu search algorithm to solve the multistage planning problem of a distribution system formulated as a multiobjective dynamic mixed integer non-linear programming problem. Multiobjective problems do not have a specific solution, but a set of solutions that allows us to observe the trade-off among the analysed objectives. Taking into account this concept, the objective functions of the model proposed in this study are: costs (investment and operational) and reliability. The actions deemed in this model for each period of the planning horizon are: increase in the capacity of existing substations (or construction of new ones), exchange of cables in existing lines (and construction of new feeders), reconfiguration of the network, allocation of sectionalising switches and construction of tie lines. The system's reliability is evaluated by means of the non-supplied energy under contingencies using the $n-1$ criterion. By line switching and the use of tie lines, part of the loads affected by a contingency can be restored, thus, the non-supplied energy can be evaluated by solving a distribution network restoration problem. Numerical results are presented for a 54-bus system.
\end{abstract}

\section{Nomenclature}

\section{Sets and Indexes}

C

$L e_{p}$
$L p_{p}$
$n A l_{n, p}$
$n b_{p}$
$n b_{p}^{S \operatorname{Sex}_{n}}$
$n l o$
$n l_{p}^{S \operatorname{Sex}_{n}}$

$n \mathrm{Sw}$

ny $\quad$ Years of the planning horizon

pp Set of periods of the planning horizon

$n \mathrm{PE} \quad$ Number of periods of the planning horizon

$\operatorname{SSex}_{p} \quad$ Set of existing substations in period $p$

$S_{S p r} \quad$ Set of proposed substations in period $p$

$n U_{j}, n, p \quad$ Set of sections of feeder $j$ of substation $n$ in period $p$

$n$ sres $\quad$ Set of restored sections

nsoff Set of offline sections

\section{Functions}

$P_{i, p}^{k}\left(V, \theta, x^{\mathrm{bop}}\right)$

Active power injection at bus $i$ with load level $k$ in period $p$
$Q_{i, p}^{k}\left(V, \theta, x^{\mathrm{bop}}\right)$

Reactive power injection at bus $i$ with load level $k$ in period $p$

Costs

$\mathrm{BCC}_{k} \quad$ Costs to build a new branch with a cable of type $k$; in $\$ / \mathrm{km}$

$\mathrm{BRC}_{a k} \quad$ Costs to replace a branch with a cable of type $a$ by a cable of type $k$; in $\$ / \mathrm{km}$

$\mathrm{SEC}_{n} \quad$ Costs to expand substation $n$; in $\$$

$\mathrm{SCC}_{n} \quad$ Costs to build a new substation $n$; in $\$$

$\mathrm{SwC}_{i j} \quad$ Costs to install a switch in line $i j$; in $\$$

$K e_{k, p} \quad$ Costs of losses with load level $k$ in period $p$; in $\$ / \mathrm{kWh}$

\section{Binary variables}

$x_{i j, k, p}^{b c} \quad$ Decision to build (1) or not (0) branch $i j$ with a cable of type $k$ in period $p$

$x_{i j, k, p}^{b r} \quad$ Decision to replace (1) or not (0) the cable of branch $i j$ by a cable of type $k$ in period $p$

$x_{n, p}^{s c} \quad$ Decision to build (1) or not (0) substation $n$ in period $p$

$x_{n, p}^{s r} \quad$ Decision variable to expand (1) or not (0) substation $n$ in period $p$

$x_{n, p}^{s o p} \quad$ Decision to operate (1) or not (0) substation $n$ in period $p$

$x_{i j, p}^{b o p} \quad$ Decision to use (1) or not (0) branch $i j$ in period $p$ Decision to install (1) or not (0) a switch in line $i j$ in period $p$ 
Continuous variables and their limits

$V_{i, k, p} \quad$ Voltage at bus $i$ with load level $k$ in period $p$

$V_{\max } \quad$ Maximum voltage level of the feeder

$V_{\text {min }} \quad$ Minimum voltage level of the feeder

$I_{i j, k, p} \quad$ Current of branch $i j$ in load level $k$ in period $p$

$I_{i j, p}^{\max } \quad$ Maximum current allowed through branch $i j$ in

period $p$

$P g_{i, p}^{k} \quad$ Active power generated at bus $i$ with load level $k$ in period $p$

$P d_{i, p}^{k} \quad$ Active power demand at bus $i$ with load level $k$ in period $p$

$Q g_{i, p}^{k} \quad$ Reactive power generated at bus $i$ with load level $k$ in period $p$

$Q d_{i, p}^{k} \quad$ Reactive power demand at bus $i$ with load level $k$ in period $p$

$S_{S \operatorname{Sex}_{n, p}} \quad$ Capacity of existing substation $n$ in period $p$

$S_{i, k, p}^{\text {load }}$

Load at bus $i$ connected to substation $n$ with load level $k$ in period $p$

$S_{i j, k, p}^{\text {losses }} \quad$ Losses in branch $i j$ connected to substation $n$ with load level $k$ in period $p$

\section{Parameters and definitions}

$d_{i j}$
$\alpha_{p}^{\text {nnv }}$
$\alpha_{p}^{\text {ope }}$
$t x j$
$t c c a_{i j}, p$

Length of branch $i j$

Investment discount factor in period $p$

Operation discount factor in period $p$

Annual interest rate

Annual current growth rate in branch $i j$ in period $p$

$l g r \_\sec _{(.), p} \quad$ Annual section load growth rate in section (.) in period $p$

$T_{k} \quad$ Duration in hours of each load level

$R_{i j, p} \quad$ Resistance of branch $i j$ in period $p$

$\mathrm{NSEC}_{j}, n, p \quad$ Non-supplied energy costs for feeder $j$ of substation $n$, in period $p$

$\mathrm{AIC}_{u}^{j, n, p} \quad$ Annual interruption costs for section $u$ of feeder $j$ of substation $n$, in period $p$

$\operatorname{Se} L_{u} \quad$ Length of section $u$

$\lambda_{u}$

$C P_{u}$

$\mathrm{CD} o_{u}^{\text {res }}$

$\mathrm{CD} o_{u}^{\text {off }}$

$\mathrm{LR}_{(.)}$

$\mathrm{LC}_{(.)}$

$\mathrm{LI}_{(.)}$

$\operatorname{LT}_{(.)}$

CRR

CRC

CRI
Rate of permanent faults for section $u$, in faults $/ \mathrm{km} /$ year

Non-supplied energy costs of section $u$ where the contingency has occurred

Non-supplied energy costs of sections downstream from section $u$, where the contingency has occurred, but service has

Non-supplied energy costs of sections downstream from section $u$, where the contingency has occurred, but service has not been restored

Percentage of residential loads in section (.)

Percentage of commercial loads in section (.)

Percentage of industrial loads in section (.)

Total load of section (.)

Residential non-supplied energy costs to repair one section with a permanent fault; in $\$ / \mathrm{MWh} /$ year

Commercial non-supplied energy costs to repair one section with a permanent fault; in \$/MWh/year

Industrial non-supplied energy costs to repair one section with a permanent fault; in $\$ / \mathrm{MWh} /$ year been restored
CCR

Residential non-supplied energy costs to restore one section affected by a permanent fault; in $\$ / \mathrm{MWh} /$ year

CCC Commercial non-supplied energy costs to restore one section affected by a permanent fault; in $\$ / \mathrm{MWh} /$ year

CCI Industrial non-supplied energy costs to restore one section affected by a permanent fault; in $\$ / \mathrm{MWh} /$ year

\section{Introduction}

Distribution system planning (DSP) is based on the technical and economic analysis of future operation conditions. A distribution company (DISCO) considers the existence of high investment costs of new equipment, the energy price and other factors, to find the best time to expand or reinforce their network aiming at the quality and reliability of the energy supply.

Modelling and solving the exact DSP problem can be a difficult job, because of its complexity and combinatorial nature. Over the years, many works describing DSP models have proposed several solution techniques to solve this problem and these techniques can be divided into two categories: heuristics and metaheuristics (Genetic algorithms, Tabu Search, Particle Swarm, etc.) [1-7]; and classical mathematical programming methods (Simplex, Branch and Bound, etc.) $[8,9]$.

Traditionally, the main objective of planning models is to minimise the required investments in new distribution equipment to supply energy for customers, taking into account demand forecasts, voltage drop, equipment capacity, etc. [6, 7, 10-13]. However, it is not enough to obtain a network with a minimum cost and acceptable power quality, but it is also necessary to supply power in a reliable manner. Thus, there are models considering either both reliability and costs in the same objective function [8, 14], or after an ex-post analysis [9, 15] or using other objective functions [1-5].

The DSP problem solution of a mono or multiobjective model indicates how and when the conductors' branches and substations must be reinforced, and when and where to build new substations and circuits. These actions improve energy supply quality and reliability because of the lower failure rate of new equipment. Other efficient actions can be used to improve system reliability, tie lines construction and the allocation of sectionalising switches (automatic or manual), which are used to control energy supply interruptions, arising from permanent faults or preventive maintenance of distribution system components, through restoration procedures. The sectionalising switches divide the feeder into sections (group of branches and load points among adjacent sectionalising switches) improving the system reliability indexes (system average interruption duration index (SAIDI), average system availability index (ASAI) and customer interruption duration (CID) at each load node and the expected energy not supplied (EENS) $[1-5,9])$, allowing for just the affected and downstream sections to be turned off in case of a contingency. Furthermore, by switching actions some of the affected sections can be relocated to other feeders through tie lines.

In this paper, DSP is formulated with a multiobjective and multistage dynamic model. The objective functions proposed are: (i) the investment and operation costs (to increase the capacity of existing substations or to build new ones, to exchange cables of existing branches and to build new 
circuits, to allocate sectionalising switches and to build tie lines), and (ii) reliability, expressed as the non-supplied energy costs (NSEC). The multiobjective approach is very important in solving these kinds of problems, because it prevents decision-making elements to be implicitly incorporated into the model, leaving the decision-making responsibility to planners. Multiobjective models do not provide a specific solution, but a set of solutions that allow observing clearly the trade-off among the analysed objectives.

To solve the multiobjective model proposed for the DPS problem a multiobjective tabu search (MOTS) algorithm is proposed [16], which uses dominance concepts to perform the optimisation. MOTS is a powerful technique to solve large and complex combinatorial problems such as the DSP problem that presents binary and continuous variables.

The main contributions of this paper are:

- A multistage dynamic model is proposed, which is different from [1, 5, 15]. The dynamic model is able to evaluate how the actions carried out in a particular period and the load growth throughout the periods of the planning horizon influence decision making.

- The model takes into account two important aspects: (i) investment costs and (ii) operation costs, using load levels to simulate the demand curve and reliability.

- All actions are optimised in a single step, different from $[1,5]$, because all actions considered in the model have a direct influence over both objectives.

This paper is structured as follows: Section 3 shows the mathematical formulation; Section 4 describes the solution technique; Section 5 shows the results for a 54-bus system [13]; and Section 6 draws conclusions.

\section{Mathematical model}

The DSP problem is formulated as a multiobjective mixed integer non-linear programming problem. The multiobjective model consists of two objective functions. The first one represents the investment and operational costs $\left(\mathrm{OF}_{\text {costs }}\right)$, which are determined at the beginning of each period and are represented by: the costs to exchange conductors of existing branches $\left(\mathrm{BRC}_{a k}\right)$; the installation costs of new branches $\left(\mathrm{BCC}_{k}\right.$ ); (in both cases the costs of the physical infrastructure are included); the costs to increase the capacity of an existing substation $\left(\mathrm{SEC}_{n}\right)$ or the construction of a new one $\left(\mathrm{SCC}_{n}\right)$; the costs of allocating sectionalising switches $\left(\mathrm{SwC}_{i j}\right)$ and the operational costs that correspond to the annual energy losses for each load level $\left(K e_{k, p}\right)$. The second one is related with the distribution system reliability $\left(\mathrm{OF}_{\text {reliability }}\right)$ in terms of the non-supplied energy costs $\left(\mathrm{NSEC}_{j, n, p}\right)$ [1-5], based on the topology and sectionalising switches allocated in the system. These objectives are minimised subject to a set of technical and operational constraints.

The proposed mathematical formulation is as follows

$$
\begin{aligned}
\mathrm{OF}_{\mathrm{costs}}= & \min \sum_{p \in p p}\left[\sum_{k \in C} \sum_{i j \in L e_{p}}\left(\mathrm{BRC}_{a k} \cdot d_{i j}\right) \cdot x_{i j, k, p}^{b r}\right. \\
& +\sum_{k \in C} \sum_{i j \in L p_{p}}\left(\mathrm{BCC}_{k} \cdot d_{i j}\right) \cdot x_{i j, k, p}^{b c} \\
& +\sum_{n \in S \operatorname{SSe} p}\left(\mathrm{SEC}_{n}\right) \cdot x_{n, p}^{s r}+\sum_{n \in S S p r_{p}}\left(\mathrm{SCC}_{n}\right) \cdot x_{n, p}^{s c}
\end{aligned}
$$

$$
\begin{aligned}
& \left.+\sum_{i j \in L e_{p}}\left(\mathrm{SwC}_{i j}\right) \cdot x_{i j, p}^{s w}\right] \cdot \alpha_{p}^{\mathrm{inv}} \\
& +\sum_{p \in p p} \sum_{k \in n l o} \sum_{i j \in L e_{p}} T_{k} \cdot K e_{k, p} \cdot R_{i j, p} \cdot I_{i j, k, p}^{2} \cdot x_{i j, p}^{\mathrm{bop}} \cdot \alpha_{p}^{\mathrm{ope}} \\
& \mathrm{OF}_{\text {reliability }}=\min \sum_{p \in p p} \sum_{n \in S \operatorname{SSe} x_{p}} \sum_{j \in n A l_{n, p}} \mathrm{NSEC}_{j, n, p}
\end{aligned}
$$

subject to

$$
\begin{aligned}
& P_{i, p}^{k}\left(V, \theta, x^{\mathrm{bop}}\right)-P g_{i, p}^{k}+P d_{i, p}^{k}=0 \quad \forall: i \in n b_{p}, \\
& k \in \text { nlo, } \quad p \in p p \\
& Q_{i, p}^{k}\left(V, \theta, x^{\mathrm{bop}}\right)-Q g_{i, p}^{k}+Q d_{i, p}^{k}=0 \quad \forall: i \in n b_{p}, \\
& k \in \text { nlo, } \quad p \in p p \\
& V_{\min }<V_{i, k, p}<V_{\max } \quad \forall: i \in n b_{p}, \quad k \in n l o, \\
& p \in p p \\
& I_{i j, k, p} \leq I_{i j, p}^{\max } \cdot x_{i j, p}^{\mathrm{bop}} \quad \forall: i j \in L e_{p}, \quad k \in n l o, \quad p \in p p \\
& 0 \leq \sum_{i \in n b_{p}^{S \operatorname{Sex}_{n}}} S_{i, k, p}^{\mathrm{load}}+\sum_{i j \in n l_{p}^{S \operatorname{Sex}_{n}}} S_{i j, k, p}^{\mathrm{losses}} \leq S_{S \operatorname{Sex}_{n, p}} \cdot x_{n, p}^{\mathrm{sop}} \\
& \forall: n \in \operatorname{SSex}_{p}, \quad k \in \text { nlo, } \quad p \in p p \\
& \sum_{i j \in L e_{p}} x_{i j, p}^{\mathrm{bop}}=n b_{p}-\sum_{n \in S \operatorname{Sex}_{p}} x_{n, p}^{\mathrm{sop}} \quad \forall p \in p p \\
& \sum_{k \in C} x_{i j, k, p}^{b r} \leq 1 \quad \forall: i j \in L e_{p}, \quad p \in p p \\
& \sum_{k \in C} x_{i j, k, p}^{b c} \leq 1 \quad \forall: i j \in L p_{p}, \quad p \in p p \\
& \sum_{i j \in L e_{p}} x_{i j, p}^{\mathrm{sw}} \leq n \mathrm{Sw} \quad \forall p \in p p \\
& x_{i j, k, p}^{b c}, x_{i j, k, p}^{b r}, x_{n, p}^{s c}, x_{n, p}^{s r}, x_{n, p}^{\mathrm{sop}}, x_{i j, p}^{\mathrm{bop}}, x_{i j, p}^{\mathrm{sw}} \in\{0,1\}
\end{aligned}
$$

In this paper, it is assumed that the investment in lines, sectionalising switches and substations is performed in the first year of each period. The costs of the annual energy losses are calculated yearly considering the annual load growth, and so the net present value over the planning horizon is calculated using $\alpha_{g}^{\text {inv }}$ and $\alpha_{g}^{\text {ope }}$ for the investment and operational costs, respectively. The latter values depend on the annual interest rate $(t x j)$, the annual current growth rate $\left(t c c a_{i j, p}\right)$ and the planning horizon.

$$
\begin{gathered}
\alpha_{p}^{\text {inv }}=\frac{1}{(1+t x j)^{(n y / n \mathrm{PE}) \cdot(p-1)}} \\
\alpha_{p}^{\text {ope }}=\sum_{y=1}^{n y / n \mathrm{PE}} \frac{\left(1+t c c a_{i j, p}\right)^{2 y}}{(1+t x j)^{(n y / n \mathrm{PE}) \cdot(p-1)+y}}
\end{gathered}
$$

The formulation of the non-supplied energy costs $\left(\mathrm{NSEC}_{j, n, p}\right)$ is based on [1]. To determine the value of $\operatorname{NSEC}_{j, n, p}$ the annual interruption cost (AIC) for each section of the 
system is calculated. The AIC formulation is as follows

$$
A I C_{u}^{j, n, p}=\lambda_{u} \cdot \operatorname{SeL}_{u} \cdot\left(\mathrm{CP}_{u}+\mathrm{CD} o_{u}^{\mathrm{res}}+\mathrm{CD}_{u}^{\mathrm{off}}\right)
$$

where $\mathrm{AIC}_{u}^{j, n, p}$ is a function of the rate of permanent faults $\left(\lambda_{u}\right)$, the length of the section $\left(\operatorname{Se} L_{u}\right)$, and the non-supplied energy costs of the sections affected by a permanent fault $\left(\mathrm{CP}_{u}, \mathrm{CD} o_{u}^{\text {res }}, \mathrm{CD} o_{u}^{\text {off }}\right)$.

Considering long-term contingencies (permanent faults or maintenance actions), distribution systems can operate in a restored state, where small changes in the topology are made (because it is a temporary state) to restore all or part of the load affected by contingencies, reducing the annual interruption cost. Finding the restored state is a difficult task, because several factors need to be taken into account. In this way, solving the restoration problem [17-19], the values of $\mathrm{CP} u, \mathrm{CD} o_{u}^{\mathrm{res}}, \mathrm{CD} o_{u}^{\text {off }}$ are evaluated as follows

$$
C P_{u}=\left(\mathrm{CRR} \cdot \mathrm{LR}_{u}+\mathrm{CRC} \cdot \mathrm{LC}_{u}+\mathrm{CRI} \cdot \mathrm{LI}_{u}\right) \cdot \mathrm{LT}_{u} \cdot \alpha_{u}^{\mathrm{NSEC}}
$$

$$
\begin{aligned}
& \mathrm{CD}_{u}^{\mathrm{res}} \\
& =\sum_{m \in n s \mathrm{res}}\left(\mathrm{CCR} \cdot \mathrm{LR}_{m}+\mathrm{CCC} \cdot \mathrm{LC}_{m}+\mathrm{CCI} \cdot \mathrm{LI}_{m}\right) \cdot \mathrm{LT}_{m} \cdot \alpha_{m}^{\mathrm{NSEC}}
\end{aligned}
$$

$$
\begin{aligned}
& \mathrm{CD} o_{u}^{\text {off }} \\
& =\sum_{m \in n \text { soff }}\left(\mathrm{CRR}^{\mathrm{L}} \mathrm{LR}_{m}+\mathrm{CRC} \cdot \mathrm{LC}_{m}+\mathrm{CRI} \cdot \mathrm{LI}_{m}\right) \cdot \mathrm{LT}_{m} \cdot \alpha_{m}^{\mathrm{NSEC}}
\end{aligned}
$$

where the non-supplied energy costs of the section where the fault occurred $\left(\mathrm{CP}_{u}\right)$ depend on the types of load: residential $\left(\mathrm{LR}_{u}\right)$, commercial $\left(\mathrm{LC}_{u}\right)$ or industrial $\left(\mathrm{LI}_{u}\right)$, and their respective interruption costs $(\mathrm{CRR}, \mathrm{CRC}, \mathrm{CRI})$, as well as the total load $\left(\mathrm{LT}_{u}\right)$ of the section. The same applies to the downstream sections where service has been restored $\left(\mathrm{CD} o_{u}^{\mathrm{res}}\right)$, and to the downstream sections where service has not been restored yet $\left(\mathrm{CD} o_{u}^{\text {off }}\right)$. The net present value of these costs is obtained as in (13) and (14) by means of $\alpha_{(.)}^{\mathrm{NSEC}}$, which depends on the annual interest rate $(t x j)$, the annual section load growth rate $\left(\operatorname{lgr} \_\sec _{(.), p}\right)$ and the planning horizon. Its expression is as follows

$$
\alpha_{(.)}^{\mathrm{NSEC}}=\sum_{y=1}^{n y / n \mathrm{PE}} \frac{\left(1+\lg r \cdot \mathrm{Sec}_{(.), p}\right)^{y}}{(1+t x j)^{(n y / n \mathrm{PE}) \cdot(p-1)+y}}
$$

Finally, $\mathrm{NSEC}_{j, n, p}$ is given by

$$
\mathrm{NSEC}_{j, n, p}=\sum_{u \in n U_{j, n, p}} \mathrm{AIC}_{u}^{j, n, p}
$$

The problem constraints are: active and reactive node balance (3) and (4), upper and lower voltage limits (5), branch conductors' capacity (6), substation operational capacity (7), radiality conditions $(3)(4)(8)$, the choice of just one type of cable to replace another one in an existing branch (9) or to be installed in a new branch (10), the maximum number of sectionalising switches allocated (11), and the setting of decision variables as binary ones (12). In addition to the constraints described above, the following ones are also considered:
- every element of the system (branch, substation and sectionalising switch) installed or replaced in period $p$ is present in period $p+1$;

- investment in new elements is considered only once;

- replacement branches or new branches may only be used after the corresponding investment has been made.

\section{Solution technique}

The proposed model is solved by a MOTS algorithm. This iterative method consists of finding the non-dominated solutions through a repeated neighbourhood search of a current solution (seed) $[16,20]$. To code the problem, a decimal-based codification is used, which allows us to include important design aspects in an easy and practical way, which would be hardly been considered with a binary codification [2]. The codification is based on [1, 2]; so, for each period, the codification structure is illustrated in Fig. 1.

In part I, the codification is as follows:

- 0 - Branch not constructed;

- $0<$ number $<100-$ Branches in operation. The number represents the type of cable installed in the branch;

- number $>100-$ Tie lines. The number represents the type of cable installed in the branch starting at 100. For example, $b_{n}=102$ is a tie line with a cable of type 2 .

Part II:

- 0 - Substation not constructed;

- $0<$ number - Type of substation. If $S_{n}$ is equal to $1, S_{n}$ is an existing substation; 1 represents the initial substation capacity, and 2 that the substation has to be expanded. On the other hand, if $S_{n} \in S S p r_{p}, 2$ represent that the substation has been constructed.

\section{Part III:}

- 0 - There are no sectionalising switches installed;

- 1 - There are sectionalising switches installed. Sectionalising switches are installed only in constructed branches.

For $n$ periods the structure of Fig. 1 is repeated $n$ times. From the system characteristics provided by the coding structure (topology, location of substations, type of cables that make up the branches, location of sectionalising switches and tie lines), a radial power flow algorithm [21] is used to calculate the system operation state, making it possible to evaluate $\mathrm{OF}_{\text {costs }}$. When working with several periods, the elements built in period $p$ must be present in period $p+1$, whether they are in operation or not. Considering this constraint, this becomes very simple through the encoding structure proposed. When a neighbour is generated through changes in period, these are passed on to the next periods. Fig. 2 illustrates this mechanism.

Fig. $2 b$ illustrates a neighbour with two modifications in period $p$, which are passed on to period $p+1$. First, the

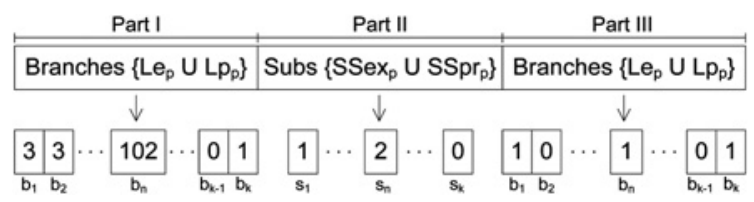

Fig. 1 Codification system for period $p$ 
position $b_{k-1}$ is built with a cable of type 1 . Since the codification presents a radial topology, inserting branch $b_{k-1}$ in period $p+1$ would create a loop. So, branch $b_{k-1}$ is inserted as a tie line in period $p+1$. Another modification is the $s_{1}$ expansion, which is passed on to period $p+1$.

Despite the fact that the planned network is meshed, its operation is radial. The tie lines along with the sectionalising switches improve the network reliability through the restoration of loads in long-term contingencies. Therefore with this information provided by the codification and having solved the restoration problem [17-19], $\mathrm{OF}_{\text {reliability }}$ can be evaluated.

MOTS is a powerful metaheuristic to solve combinatorial problems, but their efficiency is directly related to the proposed neighbourhood. In this paper, the neighbourhood for the DSP problem is defined through the simplest movements, which should consider two important conditions: connectivity of busses and radiality of the distribution network [13]. The neighbourhood movements are the following ones:

\section{i. System reconfiguration using a branch exchange technique} [22].

ii. Elimination or addition of branches (or tie lines) that supply power to a system node. If the branch added or removed is not a tie line, radiality and connectivity must be verified.

iii. Change of the power capacity of a substation in a system node; change the size of branches (or tie lines) that supply power to a system node.

iv. Elimination or addition of a substation in a system node. Eliminating a substation entails the elimination of all feeders that leave the substation and the addition of new branches to transfer the load of these feeders to other substation feeders. Adding a substation entails the creation of feeders connected to the new substation maintaining system radiality.

v. Elimination or addition of sectionalising switches in the system.

vi. Change of the positions of existing sectionalising switches in the system.

In addition to the tabu list (TL) present in classical tabu search [20], two new lists are incorporated in MOTS to solve the DSP model proposed in this paper [16]. The first one is the Pareto list (PL) that stores the non-dominated solutions found by the algorithm during the search process. The second one is the Candidate List (CL) which stores all other non-dominated solutions that have not been stored in PL and that were not explored until the current iteration. These solutions can be selected to be the new seed solution, that is, their neighbourhoods will be explored if they keep their status as non-dominated solutions during the iterative process of MOTS [16].

In the same way as the classical tabu search, a neighbourhood is generated from a current solution (seed), which is sorted into dominated and non-dominated solutions. One of the non-dominated solutions is selected to be the new seed solution, from which the search will continue. It is an intensification process that performs the search in a specific region of the search space. On the other hand, a diversification process is applied when non-dominated solutions are not found in the current neighbourhood, selecting the new seed solution from the CL. The search restarts from this solution to search a new region of the Pareto frontier, thus avoiding the premature termination of the search process because of the existence of regions with local optima.

The convergence criteria applied in the MOTS implemented in this paper is either the maximum number of iterations or until no more non-dominated solutions are found and $\mathrm{CL}=\varnothing$. The MOTS algorithm is described in Fig. 3.

\section{Results}

The implemented algorithm is tested on a 54-bus system based on [12]. This system is composed of two existing substations that can be expanded, two proposed substations, 16 existing branches and 45 proposed ones, operating at $13.8 \mathrm{kV}$. The initial system is illustrated in Fig. 4 and the system data are described in Appendix 1. Six types of cables are considered for the new and existing line expansions. Data regarding cables are given in Tables 1 and 2. Substation data are presented in Table 3.

In relation to the cable costs, positions $(0, Y), Y=1, \ldots, 6$ in Table 2, indicate the branch construction costs with a cable of type $Y$, and positions $(X, Y), X=1, \ldots, 5$ and $Y=1, \ldots, 6$, indicate the cable replacement costs of a branch with cable $X$ for cable $Y$.

The planning horizon is 15 years subdivided into three periods of 5 years. This consideration is used because of the high investment costs of the long-term planning elements, requiring a longer period to check investment return. Despite the actions being taken every 5 years, an annual load growth for each node is considered (see Appendix 1).

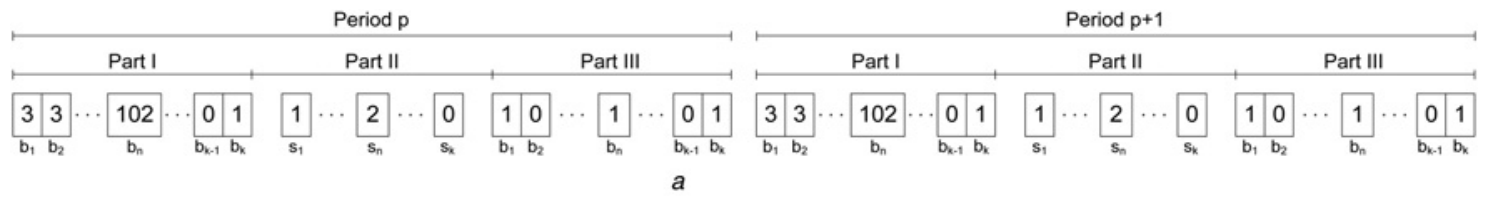

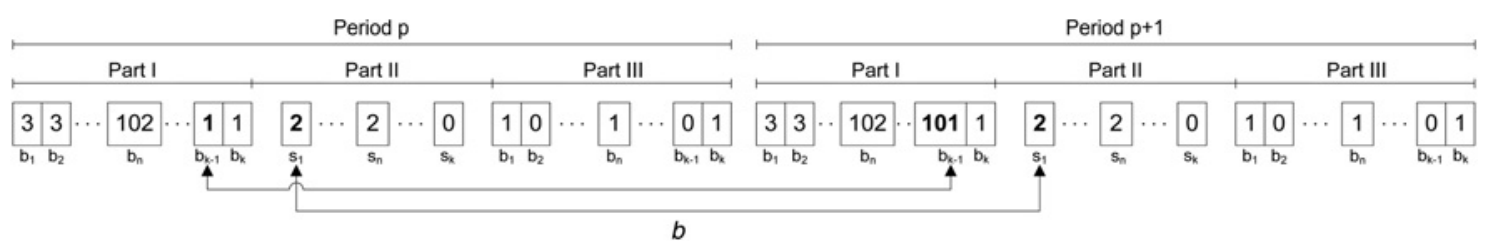

Fig. 2 Codification system for periods $p$ and $p+1$

$a$ seed solution

$b$ neighbour 


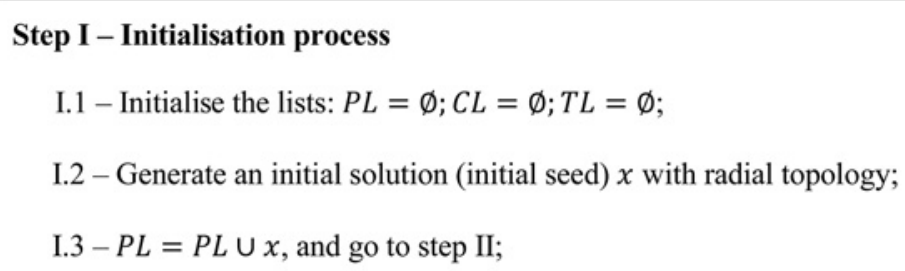

Step II - Generate and sort the neighbourhood

II. 1 - From seed $x$ generate the neighbourhood $N(x)$;

II. 2 - Sort $N(x)$ in dominated and non-dominated solutions;

II.3 - Do $\bar{N}(x)=\left\{x^{\prime} \in \bar{N}(x)\right.$ if $x^{\prime}$ is a non-dominated solution of $N(x)$ and $x^{\prime}$ does not share the stored attributes in $T L\}$, and go to step III;

\section{Step III - Find the candidate solutions}

III.1 - Eliminate from $\bar{N}(x)$ the solutions dominated by the solutions stored in $P L$ and $C L$. Then, the set of candidate solutions is $C=\{\bar{N}(x)-$ solutions dominated by the solutions stored in $P L$ and $C L\}$. Go to step IV;

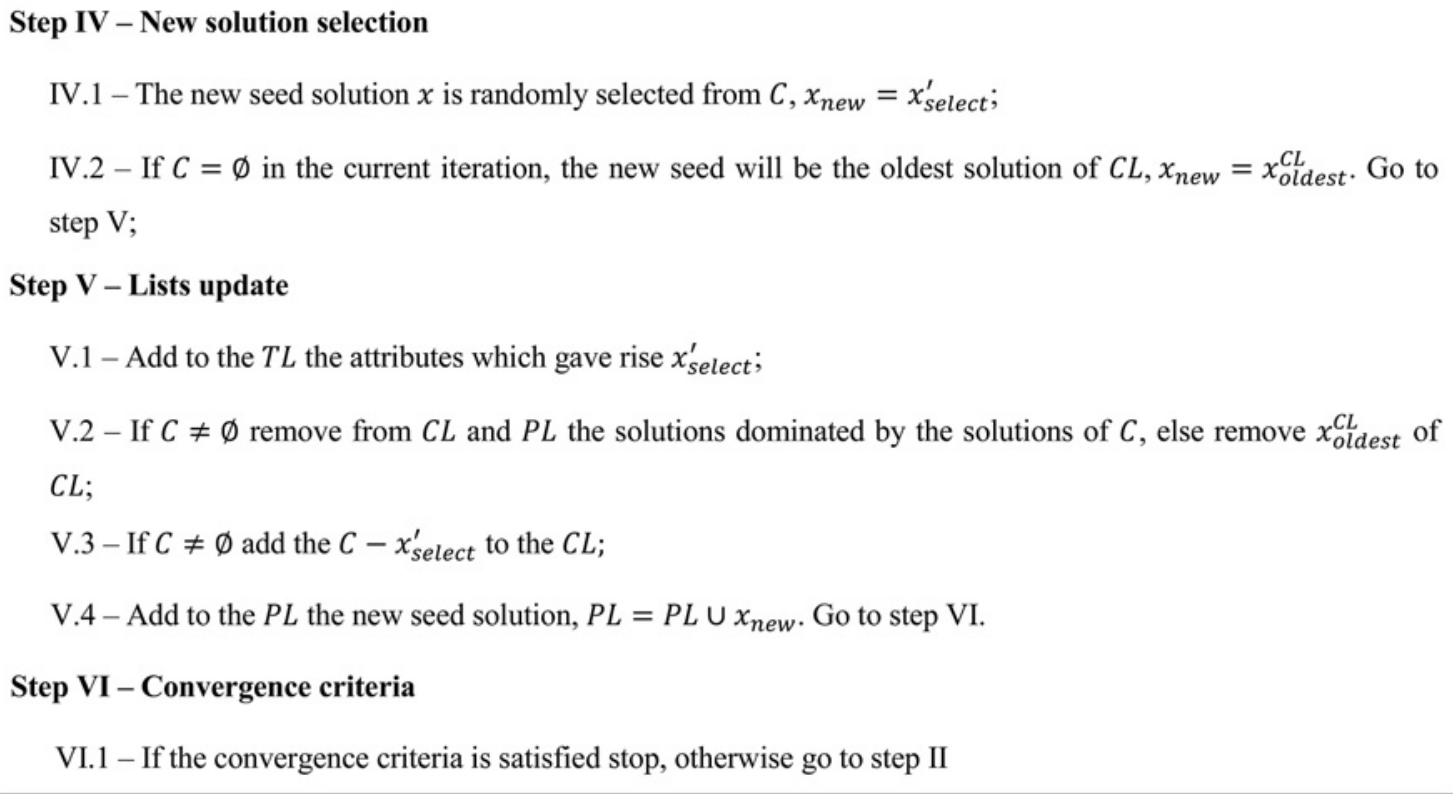

Fig. 3 MOTS algorithm

To represent the daily load curve, three load levels are used in the tests: Light - a loading factor of 0.65 with duration of $2000 \mathrm{~h} /$ year; Nominal - a loading factor of 0.80 and duration of $5760 \mathrm{~h} /$ year; and Peak - with a loading factor of 1.0 and duration of $1000 \mathrm{~h} /$ year. The upper and lower voltage limits are 0.95 and $1.05 \mathrm{pu}$, and the voltage at the substation is fixed to $1.0 \mathrm{pu}$. The energy prices to evaluate losses are $\$ 51, \$ 83$ and $\$ 100 / \mathrm{MWh}$ for light, nominal and peak levels, respectively. The costs of the sectionalising switches are $\$ 1.00 \times 10^{3}$.

The annual interest rate is $10 \%$. The rate of permanent faults is 0.4 faults $/ \mathrm{km} /$ year. The values of the interruption costs for each customer category and the interruption duration can be seen in Table 4. These values are proportional to the ones in [9].

The Pareto frontier obtained with the implemented algorithm is shown in Fig. 5. To verify the quality of the solutions found by MOTS and the trade-off between the objectives analysed, the solutions A, B and C depicted in Fig. 2 are shown in Table 5.

We can deduce from the results presented in Table 5 that planning actions influence the increase or decrease in reliability costs and total costs. For example, in solution $C$, an increased investment in construction and expansion of substations provides a better division of system loads, requiring less investment in cables, reducing losses and improving reliability costs. However, it has higher total costs due to the costs related to substations. Solution $B$ has more investments in substations, cables (tie lines) and switches with respect to solution $A$. These investments produce a greater ability to restore loads.

From an economic perspective, NESC reduction may be considered as a benefit to the DISCO, since network investments reduce payments for the energy not supplied. One analysis that can help a decision maker to decide on the best solution is to check what benefit the DISCO would 


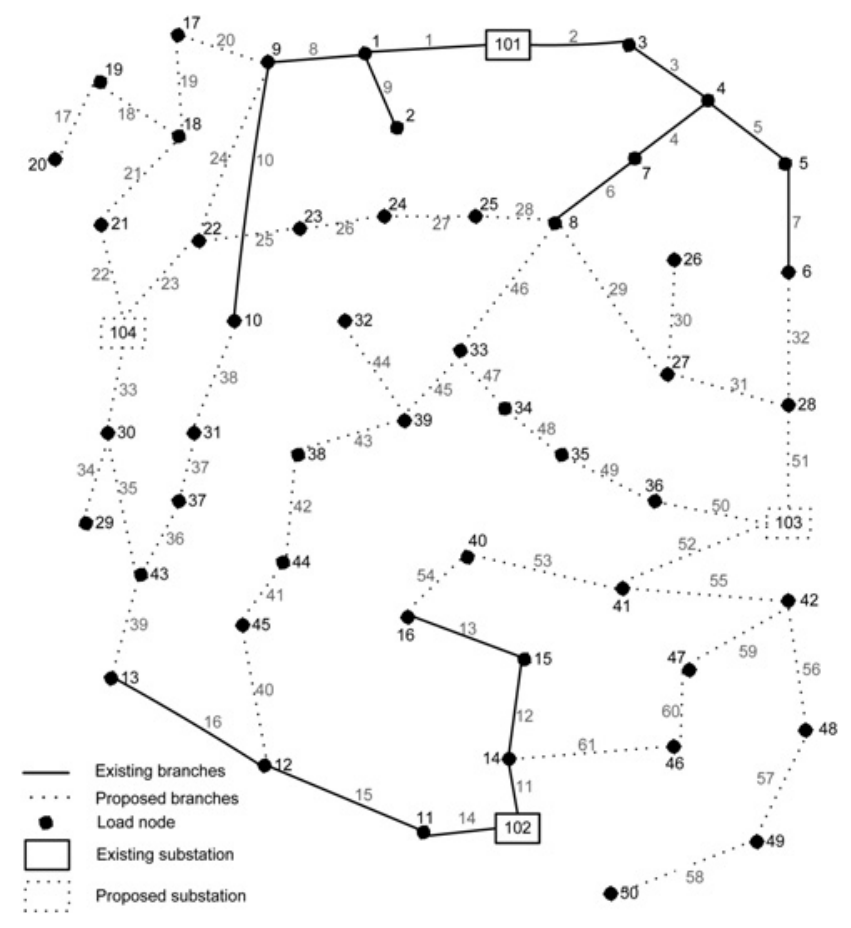

Fig. 4 Initial system

Table 1 Cable data

\begin{tabular}{lcc}
\hline Type & Current capacity (A) & Impedance $(\Omega / \mathrm{km})$ \\
\hline 1 & 150 & $0.3655+\mathrm{j} 0.2520$ \\
2 & 250 & $0.2359+\mathrm{j} 0.2402$ \\
6 & 350 & $0.1827+\mathrm{j} 0.1260$ \\
4 & 400 & $0.1460+\mathrm{j} 0.1233$ \\
5 & 500 & $0.1180+\mathrm{j} 0.1201$ \\
6 & 600 & $0.9660+\mathrm{j} 0.1201$ \\
\hline
\end{tabular}

Table 2 Cost to build and replace branches $\left(10^{3} \$ / \mathrm{km}\right)$

\begin{tabular}{lcccccc}
\hline & \multicolumn{7}{c}{ Type of cables } \\
\cline { 2 - 7 },$Y$ & 1 & 2 & 3 & 4 & 5 & 6 \\
\hline 0 & 20.0 & 30.0 & 42.0 & 50.0 & 67.0 & 85.0 \\
1 & - & 23.0 & 37.0 & 40.0 & 58.0 & 76.0 \\
2 & - & - & 30.0 & 35.0 & 50.0 & 66.0 \\
3 & - & - & - & 29.0 & 43.0 & 53.0 \\
4 & - & - & - & - & 35.0 & 45.0 \\
5 & - & - & - & - & - & 38.0 \\
\hline
\end{tabular}

Table 3 Substation data

\begin{tabular}{lcccc}
\hline Bus & $\begin{array}{c}\text { Initial } \\
\text { capacity } \\
\text { (MVA) }\end{array}$ & $\begin{array}{c}\text { New } \\
\text { capacity } \\
\text { (MVA) }\end{array}$ & $\begin{array}{c}\text { Construction } \\
\text { costs } \\
\left(10^{3} \$ / \mathrm{km}\right)\end{array}$ & $\begin{array}{c}\text { Expansion } \\
\text { costs } \\
\left(10^{3} \$ / \mathrm{km}\right)\end{array}$ \\
\hline Existing & & & & \\
101 & 16.7 & 33.4 & - & 1400.0 \\
102 & 16.7 & 30.0 & - & 1200.0 \\
New & & 22.2 & 3000.0 & - \\
103 & - & 22.2 & 3000.0 & - \\
104 & - & & & \\
\hline
\end{tabular}

obtain from the investments made in the system. Considering the solutions presented in Table 5 we have: comparing solutions $A$ and $C$, solution $C$ presents an investment cost
Table 4 Interruption costs

\begin{tabular}{lcc}
\hline Customer category & \multicolumn{2}{c}{ Interruption costs (\$/MW/year) } \\
\cline { 2 - 3 } & Restoration (15 min) & Repair (120 min) \\
\hline residential & 40.0 & 2000.0 \\
commercial & 1200.0 & 12800.0 \\
industrial & 1600.0 & 10400.0 \\
\hline
\end{tabular}

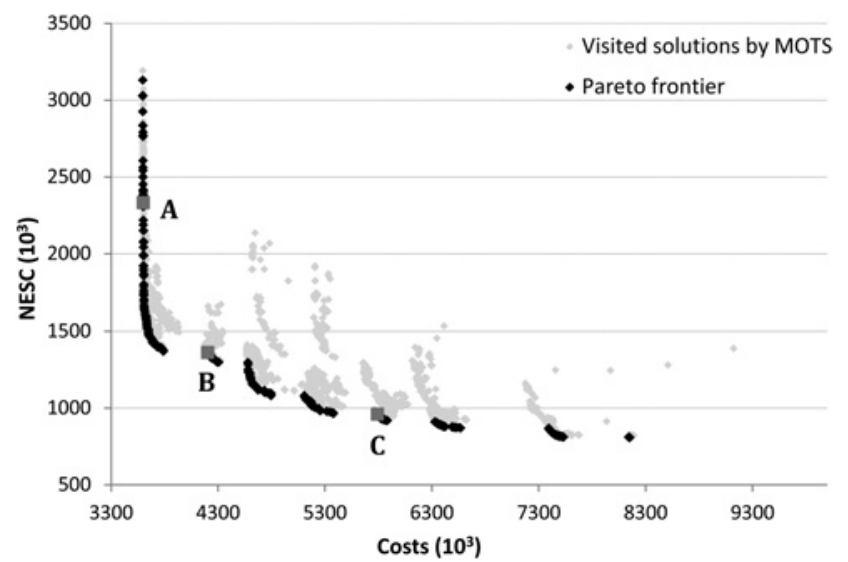

Fig. 5 Pareto frontier solutions

Table 5 Costs of solutions $A, B$ and $C$ present on the Pareto frontier

\begin{tabular}{lrrr}
\hline Parameters & \multicolumn{3}{c}{ Solution costs $\left(\$ 10^{3}\right)$} \\
\cline { 2 - 4 } & \multicolumn{1}{c}{$A$} & \multicolumn{1}{c}{$B$} & \multicolumn{1}{c}{$C$} \\
\hline losses & 877.85 & 885.23 & 648.24 \\
substation & 1862.76 & 2325.41 & 4156.63 \\
cables & 850.90 & 966.97 & 959.41 \\
switches & 9.41 & 24.61 & 26.22 \\
total investment & 2723.07 & 3316.99 & 5142.27 \\
total (inv. + losses) & 3600.92 & 4202.22 & 5790.51 \\
NSEC & 2333.08 & 1362.91 & 961.68 \\
\hline
\end{tabular}

which is $\$ 2419.2 \times 10^{3}$ higher than solution $A$, and solution $C$ presents an NSEC which is $\$ 1371.40$ smaller than the NSEC of solution $A$. The increased investment between solutions $A$ and $C$ is not offset by NSEC reduction, suggesting that solution $\mathrm{A}$ is more attractive than solution $\mathrm{C}$ from an economic viewpoint. Now, comparing solutions $A$ and $B$, solution $B$ has an investment cost $\$ 593.92 \times 10^{3}$ higher than solution $A$ and the difference in NESC of solutions $\mathrm{A}$ and $\mathrm{B}$ is $\$ 970.17 \times 10^{3}$. In this case, the investment is offset by the benefit achieved by NESC reduction, making solution $B$ more attractive than solution $A$.

The topologies for each period of the planning horizon (Figs 6-8) and the investments in each period (Table 6) are shown for solution $B$ to illustrate the characteristics of the obtained results. The costs of Table 6 are shown as net present values.

Some important observations can be inferred from the results. The first one is the reduction of the sections of the cables in branches 4-7, 7-8 and 9-22 in period 1 . When all periods are observed at once, we can observe that expansion investment in downstream branches is avoided. 


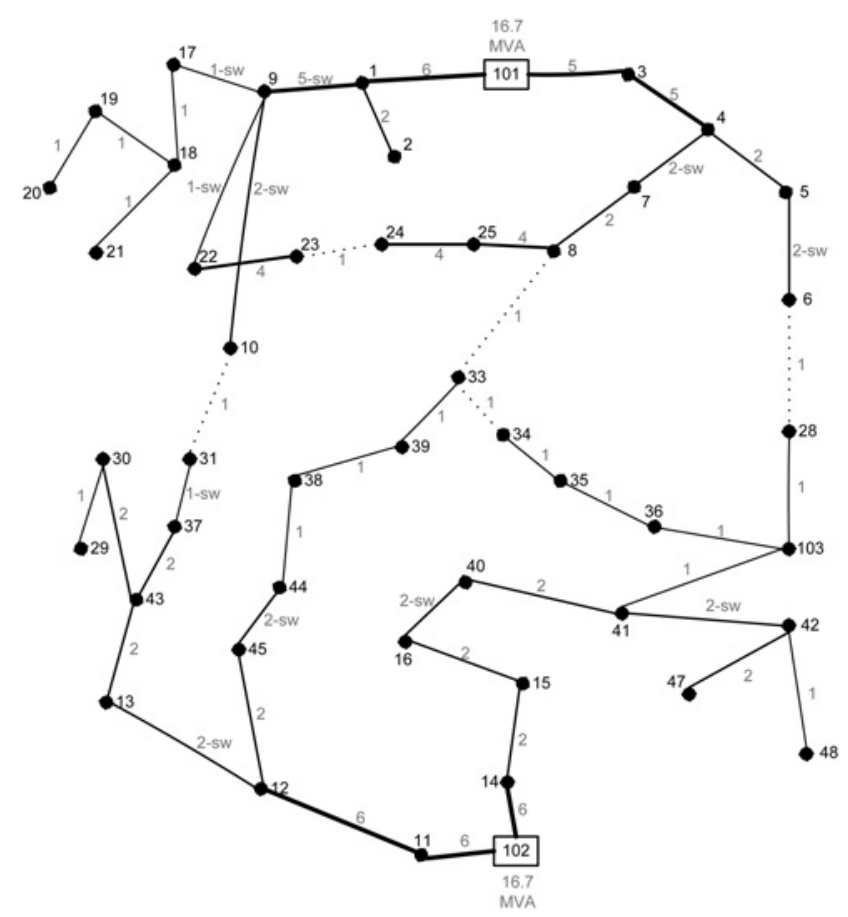

Fig. 6 Proposed solution for period 1

The second one is that tie lines and switches may be used in system reconfiguration to reduce losses, as occurs between periods 2 and 3 . However, since losses and reliability are considered in the model (in different objective functions), and system reconfiguration is taken into account in the solution technique, different topologies can be evaluated considering the same investment, though each one could have different values of reliability and losses. Thus, if these solutions are not dominated with respect to each other, these solutions are present in the Pareto frontier.

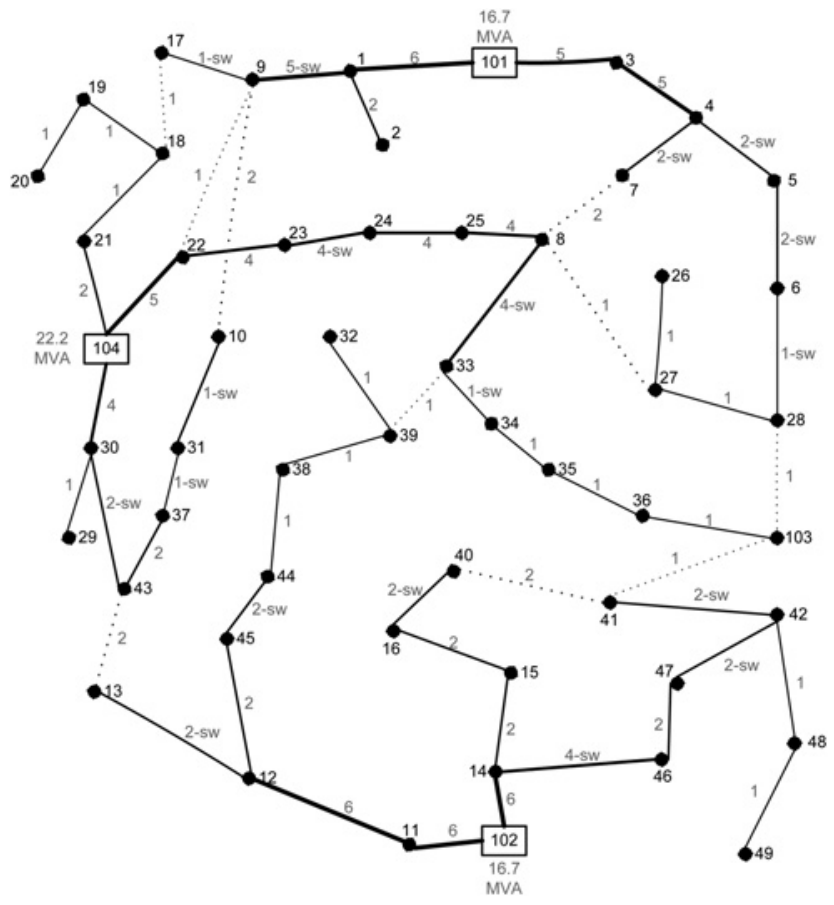

Fig. 7 Proposed solution for period 2

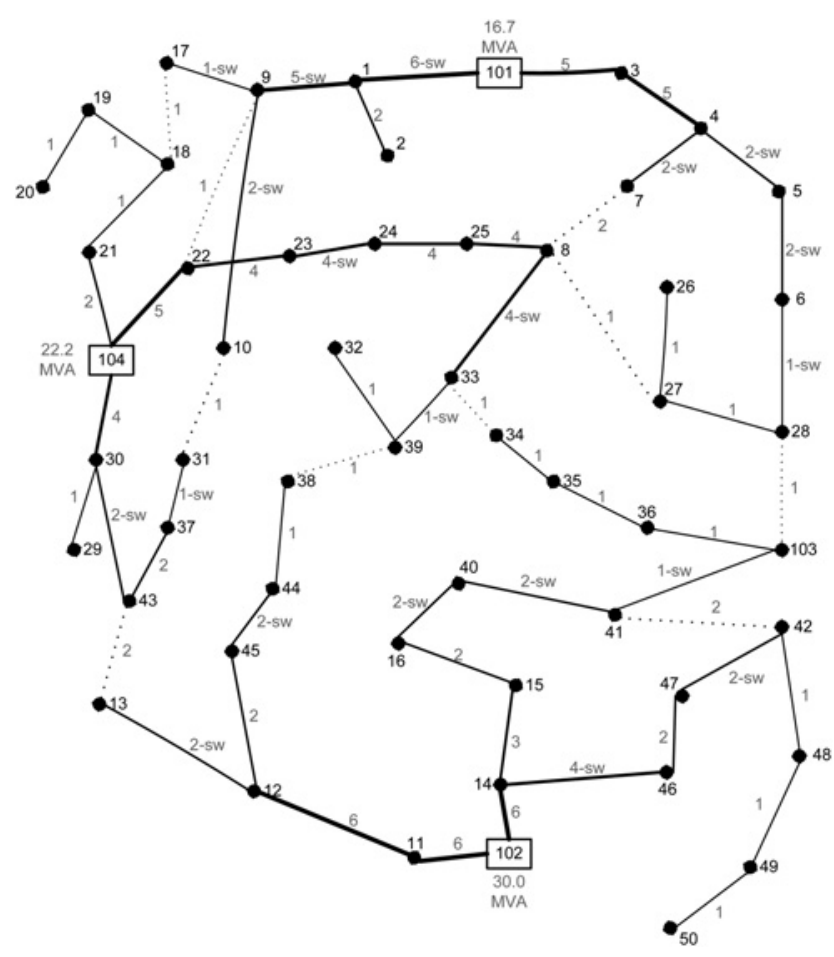

Fig. 8 Proposed solution for period 3

Table 6 investments and minimum voltage in each period for solution $b$

\begin{tabular}{lrrr}
\hline Parameters & \multicolumn{3}{c}{ Costs $\left(10^{3} \$\right)$} \\
\cline { 2 - 4 } & Period 1 & Period 2 & Period 3 \\
\hline losses & 352.74 & 247.66 & 284.83 \\
substation & 0.00 & 1862.76 & 462.65 \\
cables & 781.87 & 173.07 & 12.04 \\
switches & 16.00 & 7.45 & 1.15 \\
NSEC & 677.16 & 318.65 & 367.10 \\
$V_{\text {min }}($ pu) & 0.9771 & 0.9773 & 0.9680 \\
\hline
\end{tabular}

\section{Conclusions}

In this paper a DSP problem is formulated as a multiobjective and multistage optimisation model. The multiobjective nature of the DSP problem is related with the increase and reduction of costs to obtain a reliable network. The objective functions considered in this model take into account the investment and operational costs and reliability, under physical and technical constraints. A MOTS algorithm is used to solve the proposed model.

The proposed MOTS algorithm is very efficient to treat the multiobjective nature of the DSP problem. Through the set of solutions found by the algorithm it is possible to check the compromise (trade-off) between costs (investment and operational) and reliability of the planned network.

Beyond finding solutions that express the trade-off between the objective functions, the dynamic multistage model allows for a more comprehensive analysis of the investments made during the planning horizon. As noted in the results, some investments can be avoided and others postponed providing a lower cost of expansion.

In this way the results obtained with the model and the solution technique proposed can help a system planner (decision-maker) to select the best possible decision. 


\section{Acknowledgments}

The authors gratefully acknowledge FAPESP (grant no. 2009/ 08428-4), CNPq (grant no. 305371/2012-6) and CAPES (grant no. BEX 0144/12-6) for their economic support.

\section{References}

1 Cossi, A.M., da Silva, L.G.W., Lázaro, R.A.R., Mantovani, J.R.S.: 'Primary power distribution systems planning taking into account reliability, operation and expansion costs', IET Gener. Transm. Distrib., 2012, 6, (3), pp. 274-284

2 Ramírez-Rosado, I.J., Bernal-Agustín, J.L.: 'Reliability and costs optimization networks expansion using an evolutionary algorithm', IEEE Trans. Power Syst., 2001, 16, (1), pp. 111-118

3 Ramírez-Rosado, I.J., Domínguez-Navarro, J.A.: 'New multiobjective tabu search algorithm for fuzzy optimal planning of power distribution systems', IEEE Trans. Power Syst., 2006, 21, (1), pp. 224-233

4 Mendoza, F., Bernal-Agustín, J.L., Domínguez-Navarro, A.: 'NSGA and SPEA applied to multiobjective design of power distribution systems', IEEE Trans. Power Syst., 2006, 21, (4), pp. 1938-1945

5 Sahoo, N.C., Ganguly, S., Das, D.: 'Multi-objective planning of electrical distribution systems incorporating sectionalizing switches and tie-lines using particle swarm optimization', Swarm Evol. Comput., 2012, 3, pp. 15-32

6 Gómez, J.F., Khodr, H.M., De Oliveira, P.M., Yusta, J.M., Villasana, R., Urdaneta, A.J.: 'Ant colony system algorithm for the planning of primary distribution circuits', IEEE Trans. Power Syst., 2004, 19, (2), pp. 996-1004

7 Ramírez-Rosado, I.J., Bernal-Agustín, J.L.: 'Genetic algorithms applied to design of large power distributions systems', IEEE Trans. Power Syst., 1998, 13, (2), pp. 696-703

8 Haffner, S., Pereira, L.F.A., Pereira, L.A., Barreto, L.S.: 'Multistage model for distribution expansion planning with distributed generation -Part I: Problem Formulation', IEEE Trans. Power Syst., 2008, 23, (2), pp. 915-923

9 Lotero, R.C., Contreras, J.: 'Distribution system planning with reliability', IEEE Trans. Power Deliv., 2011, 26, (4), pp. 2552-2562

10 Gönen, T.: 'Electric power distribution system engineering' (McGraw-Hill, 1986)

11 Khator, S.R., Leung, L.C.: 'Power distribution planning: a review of models and issues', IEEE Trans. Power Syst., 1997, 12, (3), pp. $1151-1158$

12 Gönen, T., Ramírez-Rosado, I.J.: 'Review of distribution systems planning models: a model for multistage planning', IEE Proc. Gener. Distrib., 1986, 133, (9), pp. 397-408
13 Lavorato, M., Rider, M.J., Garcia, A.V., Romero, R.: 'A constructive heuristic algorithm for distribution system planning', IEEE Trans. Power Syst., 2010, 25, (3), pp. 1734-1742

14 Miranda, V., Ranito, J.V., Proença, L.M.: 'Genetic algorithms in optimal multistage distribution network planning', IEEE Trans. Power Syst., 1994, 9, (4), pp. 1927-1933

15 Samui, A., Samantaray, S.R., Panda, G.: 'Distribution system planning considering reliable feeder routing', IET Gener. Transm. Distrib., 2012, 6, (6), pp. 503-514

16 Baykasoglu, A., Owen, S., Gindy, N.: 'A taboo search based approach to find the Pareto optimal set in multiple objective optimization', Overs. Publish. Assoc., 1999, 31, (6), pp. 731-748

17 Teng, J.H., Lu, C.N.: 'Feeder-switch relocation for customer interruption cost minimization', IEEE Trans. Power Deliv., 2002, 17, (1), pp. 254-259

18 Toune, S., Fudo, H., Genji, T., Fukuyama, Y., Nakanishi, Y.: 'Comparative study of modern heuristic algorithms to service restoration in distribution systems', IEEE Trans. Power Syst., 2002, 17, (1), pp. 173-181

19 Mathias-Neto, W.P., Leão, F.B., Mantovani, J.R.S.: 'Distribution system restoration in a DG environment using a heuristic constructive multi-start algorithm'. Proc. Transmission and Distribution Conf. and Exposition: Latin America (T\&D-LA), 2010 IEEE/PES, 2010, pp. 86-91

20 Glover, F.: 'Tabu search fundamentals and uses' (University of Colorado, Bolder, CO, 1995)

21 Shirmohammadi, D.A., Hong, H.W., Semlyen, A., Luo, G.X. 'A compensation-based power flow method for weakly meshed distribution and transmission networks', IEEE Trans. Power Syst., 1988, 3, (2), pp. 753-762

22 Goswami, S.K.: 'Distribution system planning using branch exchange technique', IEEE Trans. Power Syst., 1997, 12, (2), pp. 718-723

\section{Appendix}

See Tables 7-9

\subsection{Appendix 1 -Test system data}

In Table 8, customer category represents the percentage of each category of consumers: residential, commercial and industrial. For this test system, the percentages remain the same for all periods. The values of active and reactive power correspond to the last year of the period.

Table 7 Branch data

\begin{tabular}{|c|c|c|c|c|c|c|c|c|}
\hline Branch & Cable & Length, m & Branch & Cable & Length, m & Branch & Cable & Length, m \\
\hline 1 & 2 & 562.00 & 22 & 0 & 500.00 & 43 & 0 & 686.00 \\
\hline 2 & 2 & 436.00 & 23 & 0 & 750.00 & 44 & 0 & 812.00 \\
\hline 3 & 2 & 624.00 & 24 & 0 & 936.00 & 45 & 0 & 562.00 \\
\hline 4 & 2 & 500.00 & 25 & 0 & 686.00 & 46 & 0 & 936.00 \\
\hline 5 & 2 & 624.00 & 26 & 0 & 562.00 & 47 & 0 & 374.00 \\
\hline 6 & 2 & 624.00 & 27 & 0 & 436.00 & 48 & 0 & 436.00 \\
\hline 7 & 2 & 500.00 & 28 & 0 & 562.00 & 49 & 0 & 436.00 \\
\hline 8 & 2 & 686.00 & 29 & 0 & 750.00 & 50 & 0 & 500.00 \\
\hline 9 & 2 & 624.00 & 30 & 0 & 686.00 & 51 & 0 & 624.00 \\
\hline 10 & 2 & 1436.00 & 31 & 0 & 624.00 & 52 & 0 & 624.00 \\
\hline 11 & 2 & 750.00 & 32 & 0 & 1000.00 & 53 & 0 & 750.00 \\
\hline 12 & 2 & 750.00 & 33 & 0 & 562.00 & 54 & 0 & 500.00 \\
\hline 13 & 2 & 562.00 & 34 & 0 & 624.00 & 55 & 0 & 750.00 \\
\hline 14 & 2 & 562.00 & 35 & 0 & 812.00 & 56 & 0 & 500.00 \\
\hline 15 & 2 & 624.00 & 36 & 0 & 500.00 & 57 & 0 & 750.00 \\
\hline 16 & 2 & 874.00 & 37 & 0 & 374.00 & 58 & 0 & 436.00 \\
\hline 17 & 0 & 624.00 & 38 & 0 & 624.00 & 59 & 0 & 624.00 \\
\hline 18 & 0 & 500.00 & 39 & 0 & 750.00 & 60 & 0 & 624.00 \\
\hline 19 & 0 & 812.00 & 40 & 0 & 500.00 & 61 & 0 & 686.00 \\
\hline 20 & 0 & 860.00 & 41 & 0 & 436.00 & - & - & - \\
\hline 21 & 0 & 624.00 & 42 & 0 & 624.00 & - & - & - \\
\hline
\end{tabular}


www.ietdl.org

Table 8 Bus data

\begin{tabular}{|c|c|c|c|c|c|c|c|c|c|}
\hline \multirow[t]{2}{*}{ Node } & \multicolumn{2}{|c|}{ Period 1} & \multicolumn{2}{|c|}{ Period 2} & \multicolumn{2}{|c|}{ Period 3} & \multicolumn{3}{|c|}{ Customer category } \\
\hline & $P, \mathrm{~kW}$ & $Q, \mathrm{kVAr}$ & $P, \mathrm{~kW}$ & $Q, \mathrm{kVAr}$ & $P, \mathrm{~kW}$ & $Q, \mathrm{kVAr}$ & Res., \% & Comm., \% & Ind. \% \\
\hline 1101 & 0 & 0 & 0 & 0 & 0 & 0 & 0 & 0 & 0 \\
\hline 1102 & 0 & 0 & 0 & 0 & 0 & 0 & 0 & 0 & 0 \\
\hline 1103 & 0 & 0 & 0 & 0 & 0 & 0 & 0 & 0 & 0 \\
\hline 1104 & 0 & 0 & 0 & 0 & 0 & 0 & 0 & 0 & 0 \\
\hline 1 & 2376 & 1150.72 & 2736 & 1325.12 & 3024 & 1464.56 & 70 & 20 & 10 \\
\hline 2 & 792 & 383.6 & 936 & 453.36 & 1080 & 523.04 & 60 & 20 & 20 \\
\hline 3 & 288 & 139.52 & 360 & 174.32 & 504 & 244.08 & 80 & 20 & 0 \\
\hline 4 & 1008 & 488.16 & 1368 & 662.56 & 792 & 383.6 & 75 & 10 & 15 \\
\hline 5 & 1440 & 697.44 & 1656 & 802 & 1872 & 906.64 & 0 & 0 & 100 \\
\hline 6 & 432 & 209.2 & 504 & 244.08 & 504 & 244.08 & 65 & 25 & 10 \\
\hline 7 & 144 & 69.76 & 360 & 174.32 & 720 & 348.72 & 50 & 50 & 0 \\
\hline 8 & 1080 & 523.04 & 1224 & 592.8 & 1368 & 662.56 & 70 & 20 & 10 \\
\hline 9 & 1368 & 662.56 & 1440 & 697.44 & 864 & 418.48 & 80 & 20 & 0 \\
\hline 10 & 1440 & 697.44 & 1728 & 836.88 & 2088 & 1011.28 & 60 & 25 & 15 \\
\hline 11 & 144 & 69.76 & 216 & 104.64 & 216 & 104.64 & 70 & 30 & 0 \\
\hline 12 & 720 & 348.72 & 1152 & 557.92 & 1296 & 627.68 & 30 & 70 & 0 \\
\hline 13 & 648 & 313.84 & 720 & 348.72 & 792 & 383.6 & 70 & 20 & 10 \\
\hline 14 & 576 & 278.96 & 648 & 313.84 & 720 & 348.72 & 60 & 20 & 20 \\
\hline 15 & 720 & 348.72 & 864 & 418.48 & 1008 & 488.16 & 80 & 20 & 0 \\
\hline 16 & 936 & 453.36 & 1080 & 523.04 & 1368 & 662.56 & 75 & 10 & 15 \\
\hline 17 & 360 & 174.32 & 432 & 209.2 & 504 & 244.08 & 0 & 0 & 100 \\
\hline 18 & 648 & 313.84 & 720 & 348.72 & 864 & 418.48 & 65 & 25 & 10 \\
\hline 19 & 720 & 348.72 & 864 & 418.48 & 1008 & 488.16 & 50 & 50 & 0 \\
\hline 20 & 360 & 174.32 & 504 & 244.08 & 576 & 278.96 & 70 & 20 & 10 \\
\hline 21 & 360 & 174.32 & 1152 & 557.92 & 1296 & 627.68 & 70 & 30 & 0 \\
\hline 22 & 360 & 174.32 & 720 & 348.72 & 792 & 383.6 & 60 & 25 & 15 \\
\hline 23 & 360 & 174.32 & 648 & 313.84 & 720 & 348.72 & 70 & 20 & 10 \\
\hline 24 & 360 & 174.32 & 288 & 139.52 & 360 & 174.32 & 60 & 20 & 20 \\
\hline 25 & 432 & 209.2 & 576 & 278.96 & 648 & 313.84 & 80 & 20 & 0 \\
\hline 26 & 0 & 0 & 576 & 278.96 & 864 & 418.48 & 75 & 10 & 15 \\
\hline 27 & 0 & 0 & 864 & 418.48 & 1080 & 523.04 & 0 & 0 & 100 \\
\hline 28 & 288 & 139.52 & 360 & 174.32 & 504 & 244.08 & 45 & 55 & 0 \\
\hline 29 & 432 & 209.2 & 648 & 313.84 & 1008 & 488.16 & 50 & 50 & 0 \\
\hline 30 & 1440 & 697.44 & 1656 & 802 & 1872 & 906.64 & 70 & 30 & 0 \\
\hline 31 & 432 & 209.2 & 504 & 244.08 & 504 & 244.08 & 80 & 20 & 0 \\
\hline 32 & 0 & 0 & 1080 & 523.04 & 1224 & 592.8 & 60 & 25 & 15 \\
\hline 33 & 1296 & 627.68 & 1656 & 802 & 2088 & 1011.28 & 70 & 20 & 10 \\
\hline 34 & 648 & 313.84 & 720 & 348.72 & 864 & 418.48 & 60 & 20 & 20 \\
\hline 35 & 0 & 0 & 0 & 0 & 648 & 313.84 & 80 & 20 & 0 \\
\hline 36 & 144 & 69.76 & 144 & 69.76 & 216 & 104.64 & 75 & 10 & 15 \\
\hline 37 & 720 & 348.72 & 1152 & 557.92 & 1512 & 732.32 & 0 & 0 & 100 \\
\hline 38 & 0 & 0 & 0 & 0 & 792 & 383.6 & 65 & 25 & 10 \\
\hline 39 & 576 & 278.96 & 648 & 313.84 & 720 & 348.72 & 20 & 80 & 0 \\
\hline 40 & 720 & 348.72 & 864 & 418.48 & 1008 & 488.16 & 70 & 20 & 10 \\
\hline 41 & 216 & 104.64 & 360 & 174.32 & 648 & 313.84 & 80 & 20 & 0 \\
\hline 42 & 0 & 0 & 0 & 0 & 864 & 418.48 & 60 & 25 & 15 \\
\hline 43 & 0 & 0 & 0 & 0 & 936 & 453.36 & 70 & 20 & 10 \\
\hline 44 & 360 & 174.32 & 720 & 348.72 & 1008 & 488.16 & 60 & 20 & 20 \\
\hline 45 & 360 & 174.32 & 360 & 174.32 & 576 & 278.96 & 80 & 20 & 0 \\
\hline 46 & 0 & 0 & 1152 & 557.92 & 1296 & 627.68 & 75 & 10 & 15 \\
\hline 47 & 360 & 174.32 & 360 & 174.32 & 720 & 348.72 & 0 & 0 & 100 \\
\hline 48 & 360 & 174.32 & 432 & 209.2 & 576 & 278.96 & 65 & 25 & 10 \\
\hline 49 & 0 & 0 & 432 & 209.2 & 360 & 174.32 & 50 & 50 & 0 \\
\hline 50 & 0 & 0 & 0 & 0 & 576 & 278.96 & 70 & 20 & 10 \\
\hline
\end{tabular}

44

(C) The Institution of Engineering and Technology 2013
IET Gener. Transm. Distrib., 2014, Vol. 8, Iss. 1, pp. 35-45 doi: 10.1049/iet-gtd.2013.0115 
www.ietdl.org

Table 9 Annual load growth for each node (\%)

\begin{tabular}{|c|c|c|c|c|c|c|c|}
\hline Node & Period 1 & Period 2 & Period 3 & Node & Period 1 & Period 2 & Period 3 \\
\hline 1101 & 0 & 0 & 0 & 24 & 0.981 & -4.365 & 4.564 \\
\hline 1102 & 0 & 0 & 0 & 25 & 4.213 & 5.922 & 2.384 \\
\hline 1103 & 0 & 0 & 0 & 26 & 0 & 1.172 & 8.447 \\
\hline 1104 & 0 & 0 & 0 & 27 & 0 & 1.172 & 4.564 \\
\hline 1 & 2.445 & 2.862 & 2.022 & 28 & 5.79 & 4.564 & 6.961 \\
\hline 2 & 3.152 & 3.398 & 2.903 & 29 & 8.846 & 8.447 & 9.239 \\
\hline 3 & 5.79 & 4.564 & 6.961 & 30 & 2.659 & 2.835 & 2.482 \\
\hline 4 & 0.981 & 6.298 & -10.355 & 31 & 1.614 & 3.131 & 0 \\
\hline 5 & 2.659 & 2.835 & 2.482 & 32 & 0 & 1.172 & 2.535 \\
\hline 6 & 1.614 & 3.131 & 0 & 33 & 4.885 & 5.025 & 4.745 \\
\hline 7 & 17.608 & 20.112 & 14.87 & 34 & 2.934 & 2.13 & 3.714 \\
\hline 8 & 2.393 & 2.535 & 2.249 & 35 & 0 & 0 & 0.787 \\
\hline 9 & 0.981 & 1.031 & -9.712 & 36 & 4.564 & 0 & 8.447 \\
\hline 10 & 3.786 & 3.714 & 3.857 & 37 & 7.807 & 9.856 & 5.589 \\
\hline 11 & 4.564 & 8.447 & 0 & 38 & 0 & 0 & 0.787 \\
\hline 12 & 6.382 & 9.856 & 2.384 & 39 & 2.257 & 2.384 & 2.13 \\
\hline 13 & 2.027 & 2.13 & 1.924 & 40 & 3.424 & 3.714 & 3.131 \\
\hline 14 & 2.257 & 2.384 & 2.13 & 41 & 11.629 & 10.757 & 12.475 \\
\hline 15 & 3.424 & 3.714 & 3.131 & 42 & 0 & 0 & 1.362 \\
\hline 16 & 3.89 & 2.903 & 4.841 & 43 & 0 & 0 & 1.362 \\
\hline 17 & 3.424 & 3.714 & 3.131 & 44 & 11.196 & 14.87 & 6.961 \\
\hline 18 & 2.934 & 2.13 & 3.714 & 45 & 5.387 & 0 & 9.856 \\
\hline 19 & 3.424 & 3.714 & 3.131 & 46 & 0 & 1.172 & 2.384 \\
\hline 20 & 4.92 & 6.961 & 2.707 & 47 & 8.447 & 0 & 14.87 \\
\hline 21 & 16.679 & 26.191 & 2.384 & 48 & 4.841 & 3.714 & 5.922 \\
\hline 22 & 9.161 & 14.87 & 1.924 & 49 & 0 & 1.172 & -3.581 \\
\hline 23 & 7.797 & 12.475 & 2.13 & 50 & 0 & 0 & 0.981 \\
\hline
\end{tabular}

